\title{
A CORRECT APPROACH ON ADDING CRITERIA IN THE ANALYTIC HIERARCHY PROCESS
}

\author{
Min-Suk YOON* \\ Professor, Electronic Commerce at College of Culture \& Social Sciences \\ Chonnam National University \\ Yeosu-City, 550-749, S. Korea \\ E-mail: msyoon@chonnam.ac.kr \\ Young-Woo SOHN \\ Research Associate Professor, Creative IT Excellence Engineering \\ Pohang University of Science and Technology \\ Pohang-City, 790-784, S. Korea \\ E-mail: ywsohn@postech.ac.kr
}

\begin{abstract}
This paper deals with addition besides deletion of indifferent or wash criteria on which the alternatives have equal performance in the Analytic Hierarchy Process (AHP) against the misguided argument as shortcoming of the AHP. In such case, it is noted that careful attention and a correct approach are required in order not to be led towards a wrong decision with rank reversal when composing priorities of criteria and performances of alternatives according to the criteria.

Contrasting with the case of alternatives, prioritizing added or deleted indifferent criteria requires consistency with the change of conceptual definition in regards to the related elements as well as that of the hierarchical structure if needed. Corresponding to such importance in ranking alternatives, two principles of the AHP need to be considered simultaneously: the completeness of expectation axiom and the outer dependency (hierarchical independence) axiom on which the argument of rank reversal focuses only.

It is shown that the Analytic Network Process (ANP) as a general approach can cover the case adding feedback or inner dependency to the given hierarchic relation of criteria. Finally, this paper presents the way in which there is no irrational/unreasonable rank reversal with simple addition of indifferent criteria in addition to the deletion of wash criteria, which helps AHP/ANP application.
\end{abstract}

Keywords: Analytical Hierarchy Process; Analytical Network Process; Criteria Addition; Rank Reversal; Correct Approach

\footnotetext{
${ }^{*}$ Corresponding author
} 


\section{Introduction}

To the human mind, all physical things reveal themselves as properties and interaction of properties. Every physical object is represented by a collection of properties that can be understood in terms of their intensity as stimuli for the brain or in terms of their relativity to the same property possessed by other similar objects. Attributes are properties interested for a decision out of properties that objects have. However, both are interchangeable in the Analytic Hierarchy Process (AHP) as criteria and a primitive is criterion. To make a decision is to select an object that is expected to produce the largest amount of output. To measure the properties without including the measurement to interpret, we must use our experience and understanding to form judgments from which we then derive measurement in the form of priorities. In the AHP, these judgments are expressed quantitatively on an absolute scale that denotes dominance of one element over another so that a best outcome can be derived by combining and trading off different factors or attributes [Saaty 2010].

The AHP has contributed to decision making, especially when dealing with judgment data; it includes descriptive as well as normative orientations in ranking elements. Ranking alternatives using the AHP is basically composed of two parts; prioritizing hierarchical criteria and alternatives according to each criterion in the lowest level, and synthesizing them. The choice is based on the total sum of performances along the criteria for each alternative. The AHP has undergone debates about rank reversal concerned with the result of synthesis. Rank reversal is superficially only change on previous ranking results after adding or deleting alternatives or criteria. However specifically that is caused by both how to prioritize alternatives or criteria based on the meaning of their structure and how to synthesize them. The focus on the debate needs to be the validity of change in ranking; rank reversal is right or wrong.

The central concern in ranking by the AHP has been whether alternatives are independent or dependent on one another within the hierarchy. If the measurements of alternatives are assumed to be independent of each other, there is no rank reversal even though other alternatives are added or deleted. However considering dependability, rank reversal is caused by adding or deleting alternative [Millet \& Saaty 2000]. It is also concerned with how to prioritize alternatives by two modes; distributive and ideal AHP. The former is by comparing alternatives and the latter is by rating things with respect to a standard or ideal [Saaty \& Vargas 2006]. So, if the alternatives are dependent among themselves, then distributive mode is required and anything can happen to rank. Otherwise, rank is preserved when new alternative are added or old ones are deleted because the number assigned is unaffected by the evaluation of other alternatives. A standard or ideal case is required under each criterion, which allows priorities to exceed unity.

Recently the argument of rank reversal has been concerned with the addition or deletion of criteria in addition to that of alternatives [Finan and Hurley 2002, Perez et al. 2006]. In general, it is known in decision making that if one alters criteria or their weights then the outcome of a decision will change possibly leading to rank reversal. There are usually two situations for changing criteria. The first is 'wash criteria' which involves the deletion of criteria that are assumed to be irrelevant because the alternatives have equal priorities under them (Finan and Hurley 2002). The second is called 'indifferent criteria' which involves the addition criteria, which is also assumed to be irrelevant for the same reasons as wash criteria (Perez et al. 2006). Those authors argued that rank reversal in such cases is caused by shortcomings of the AHP. Saaty \& Vargas (2006) illustrate that dropping wash criteria is unreasonable. Using the Analytic Network Process (ANP), they showed there was the error of renormalizing the weight of the remaining criteria that rise to rank reversal in the paper regarding wash criteria. It is easy to see that small perturbations of the equal values for the alternatives with respect to an overwhelmingly important criterion can make a substantial difference to the final priorities of the alternatives if the wash criterion is blindly deleted from the set of criteria. Then it was proposed not to delete wash criteria but simply to assign zero performance values to the alternatives. For the case of indifferent criteria, Saaty et al. (2009) 
proposed not to add them or proposed to consider that a new decision respecting the influence of added criteria.

However, no study has proved the wrong process argued by Perez et al. (2006) in normalizing the influence of added criteria and leading to rank reversal. They focused on an axiom of the AHP, which assumes that upper level criteria are independent of lower level criteria in hierarchical decomposition. The related axiom is outer dependency in terms of lower criteria. Focusing on this axiom, this study suggests the proper way of adding criteria even though they are indifferent among alternatives.

In order to clarify the structure of elements, we describe the meaning of hierarchical independence or outer dependency including one more related principle concerning the hierarchy within the AHP. Then we research their meaning related to the substantial entity of the AHP for rank preservation. So, we apply those axioms to the example given by the previous study to correct the results. The renormalizing process in the AHP shows the influence of added criteria on the final outcome with no rank reversal. Sequentially, consistent concepts are generalized using the ANP and consistent output is obtained from the ANP. Finally, we correct the previous argument and conclude the addition or deletion of criteria.

\section{Outer Dependence and Completeness}

The most significant aspects of the AHP are: 1) structural representation of a complex problem hierarchy, 2) rational and scientific method to illicit subjective judgments into quantitative index pairwise comparisons and 3) using a ratio scale instead of an interval or other scales. The related axioms or principles include: hierarchical composition with outer-dependence, completeness of criteria to expect alternatives rank in addition to the reciprocal, and homogeneity axioms regarding pairwise comparison measurements.

\section{Reasons for Hierarchies}

Hierarchy represents a multilevel structure whose first level is a goal followed successively by levels of factors, criteria and subcriteria, and a bottom level of alternatives. The hierarchy enables us to make practical decisions based on a cause-and-effect understanding or judgments systematically. The hierarchy should be stable and flexible; stable in that small changes have small effect and flexible in that additions to a well-structured hierarchy do not disrupt the performance. Hierarchical representation of a system can be used to describe how changes in priority at upper levels affect the priority of elements in lower levels, and vice versa. They give great detail of information on the structure and function of a system in the lower levels and provide an overview of the actors and their purposes in the upper levels. It is possible to evaluate both top down or bottom up directions in a hierarchy [Saaty 2006].

\section{Pairwise comparisons}

Comparing elements pairwise using a ratio scale implies that the importance (or priority) of one element can only be determined in terms of the priority of the others. Pairwise comparisons can reflect dependence among elements to be compared. Evaluating elements always depends on each other in the mind in making comparisons because once identified, they are remembered and categorized and cannot be forgotten easily, and the mind inevitably compares and contrasts them whether we like it or not. Hence, the mind may prefer evaluating something depending on its experience with similar elements and thus the evaluation of that element depends on knowledge of other elements. In fact, the knowledge one has depends on all the elements that one is familiar with. Pairwise comparisons deal with the dependency among elements according to the structure and relations in mind (Miller \& Saaty 2000). Thus, it would seem that pairwise comparisons allow rank reversal and hence, is mathematically more general, and it also seems more natural than normatively preserving rank by rating alternatives (Saaty 2006). 


\section{Ratio scale}

In the AHP, the ratio scale for absolute numbers is used for pairwise comparisons of criteria and alternatives. The ratio scale can be propagated hierarchically, that is, this applies to comparing not only alternatives with respect to criteria but also to comparing criteria with respect to higher order criteria or goals. Ratio scales and hierarchies are well suited to be together. In contrast, no other kind of derived scales have this property. For example, the interval scale in utility theory cannot be used to perform arithmetic operations such as addition, multiplication and division. Additionally, ratio scale makes it possible to combine criteria and alternative measurements. However, in utility theory the importance of criteria is grounded in their use to discriminate among the alternatives. Thus, a criterion is unimportant if the alternatives have equal values or are judged to be equal under it. It is paradoxical that if the measurements regarding the alternatives are close under a very important criterion, such a criterion should be dropped from consideration in a multi-criteria decision [Saaty \& Vargas 2006].

In terms of dependency, there are two kinds: judgmental dependency and structural dependency in a hierarchy with feedback, which includes functional (input-output) dependency in the structure. Judgmental dependency can be dealt by pairwise comparisons among elements. The structural dependency of feedback is solved by the ANP, the general form of the AHP. The AHP deals with hierarchical dependency but the ANP deals with various directions of dependency among criteria, and alternatives in addition to hierarchical dependency.

Here, it is necessary to complete a re-overview of the independence axiom of the AHP because Fiana \& Hurley (2002) and Perez et al. (2006) argue that based on the axiom, the AHP causes rank reversal with addition or deletion of wash or indifferent criterion and that they are a shortcoming of the AHP, which is not correct and which motivates this study.

The independence axiom can be re-expressed as outer dependence concerned with hierarchical decomposition (Saaty, 2006). This axiom implies containment of the small elements by large clusters or levels. From this axiom, inner dependence for a hierarchy is defined as that the elements in a level may depend on one another with respect to a property in another level. Input-output dependence of industry (e.g. manufacturing) demonstrates the idea of inner dependence. To form a hierarchy, the following axiom is given.

Outer dependence Axiom:

Let $\mathrm{H}$ be a hierarchy with levels $\mathrm{L}_{1}, \mathrm{~L}_{2}, \ldots, \mathrm{L}_{\mathrm{H}}$. For each $\mathrm{L}_{\mathrm{k}}, \mathrm{k}=1,2, \ldots, \mathrm{H}-1$,

(1) $\mathrm{L}_{\mathrm{k}+1}$ is outer dependent on $\mathrm{L}_{\mathrm{k}}$,

(2) $\mathrm{L}_{\mathrm{k}}$ is not outer dependent on $\mathrm{L}_{\mathrm{k}+1}$,

(3) $L_{k+1}$ is not inner dependent with respect to any $x{ }^{E} L_{k}$.

If one is to omit this Axiom, the principle of hierarchic composition would no longer apply because of outer and inner dependence among levels or components which need not form a hierarchy. The appropriate composition principle is derived from the supermatrix approach for networks. Hierarchical composition is a special case of the network following axiom, which means the axiom with feedback:

Outer dependence Axiom with feedback:

let $C_{1}, C_{2}, \ldots C_{n}$ be the nonempty subsets of a network. For each $C_{i}$ there is some $C_{j}$ so that either $\mathrm{C}_{\mathrm{i}}$ is outer dependent on $\mathrm{C}_{\mathrm{j}}$ or $\mathrm{C}_{\mathrm{j}}$ is outer dependent on $\mathrm{C}_{\mathrm{i}}$ or both.

Note that outer dependency and network axioms need to be considered with the completeness of expectation. The purpose of hierarchical decomposition or network relation is to structure the criteria in 
order to evaluate alternatives. Then, the ranking of alternatives can be expected from the performance of alternatives according to criteria. Judgments require beliefs about the rank of alternatives derived from prior knowledge. The beliefs are expectations in the AHP and its expression is as follows,

\section{Expectation Axiom with Completeness}

(1) Completeness: $€ \subset \mathrm{H}-\mathrm{L}_{\mathrm{H}}$, where $\mathrm{L}_{\mathrm{H}}$ is a finite set of alternatives

(2) Rank: To preserve rank independently of what and how many other alternatives there may be. Alternatively, to allow rank to be influenced by the number and the measurements of alternatives that are added to or deleted from the set.

This axiom simply says that those thoughtful individuals who have reasons for their beliefs should make sure that their ideas are adequately represented for the outcome to match these expectations; i.e., all criteria are represented in the hierarchy or the network in general. Actually, expectations are not only about the structure of a decision and its completeness, but also the judgments and their redundancy to capture reality and the inconsistency that should be improved with redundancy.

A general way to cope with changing criteria items is use of the ANP. If the change requires adopting complex dependency, its solution is obtained by applying the ANP (See following two Tables). Generally, the hierarchic relation of outer dependence is generalized to the network, $W_{\mathrm{CG}}$ and $\mathrm{W}_{\mathrm{AC}}$. $\mathrm{W}_{\mathrm{CG}}$ is composed of eigenvectors of pairwise comparison matrices along with hierarchic levels. The general supermatrix of the ANP includes inner dependence among criteria $\left(\mathrm{W}_{\mathrm{CC}}\right)$ or among alternatives $\left(\mathrm{W}_{\mathrm{AA}}\right)$ in addition to feedback $\left(\mathrm{W}_{\mathrm{CA}}\right)$ that shows the characteristics of each alternative reversely to criteria evaluation. If there is no inner dependence from the general supermatrix, then only feedback from alternatives to criteria is added to hierarchic relation (Table B). The submatrix of the final ranking of alternatives in the limiting supermatrix can be represented by using $\mathrm{W}_{\mathrm{AC}}$ and $\mathrm{W}_{\mathrm{CA}}$.

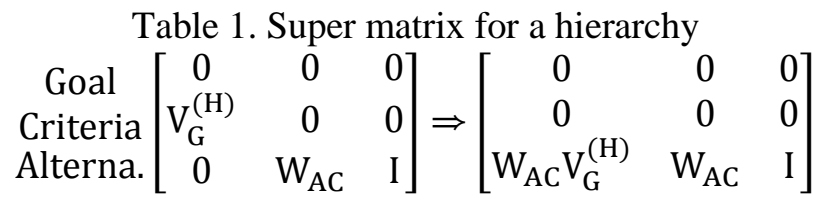

$\mathrm{V}_{\mathrm{G}}^{(\mathrm{H})}$ : the column global eigenvector of priorities of criteria at the bottom level with respect to the goal $\mathrm{W}_{\mathrm{AC}}$ : the matrix of column eigenvectors of the alternatives with respect to each criterion

Table 2. General form for a network

Goal
Criteria
Alterna. $\left[\begin{array}{ccc}0 & 0 & 0 \\ \mathrm{~W}_{\mathrm{CG}} & \mathrm{W}_{\mathrm{CC}} & \mathrm{W}_{\mathrm{CA}} \\ 0 & \mathrm{~W}_{\mathrm{AC}} & \mathrm{W}_{\mathrm{AA}}\end{array}\right]$

Table 3. With only feedback for a network

$$
\begin{gathered}
\text { Goal } \\
\text { Criteria } \\
\text { Alterna. }
\end{gathered}\left[\begin{array}{ccc}
0 & 0 & 0 \\
\mathrm{~W}_{\mathrm{CG}} & 0 & \mathrm{~W}_{\mathrm{CA}} \\
0 & \mathrm{~W}_{\mathrm{AC}} & 0
\end{array}\right]
$$

$\mathrm{W}_{\mathrm{CG}}$ : the matrix composed of column eigenvectors of the criteria with respect to upper criterion or the goal

$\mathrm{W}_{\mathrm{AC}}$ : the matrix composed of column eigenvectors of the alternatives with respect to each criterion $\mathrm{W}_{\mathrm{CA}}$ : the matrix composed of column eigenvectors of the criteria with respect to each alternative $\mathrm{W}_{\mathrm{CC}}$ : the matrix composed of column eigenvectors of inner dependence among the criteria $\mathrm{W}_{\mathrm{AA}}$ : the matrix composed of column eigenvectors of inner dependence among the alternatives 


\section{Addition of Indifference Criteria}

\subsection{Incorrect Example}

This paper points and corrects misunderstanding of AHP principles in the given example of rank reversal by adding an indifferent criterion under which alternatives are equal from Perez et al. (2006). It should be noted that the example dealt with the 'distributive mode' of the AHP.

The example is given as Part I and Part II in Table 1. Let $\mathrm{C}$ and $\mathrm{C}^{\prime}$ be the criteria for global aim. Let $\{\mathrm{C} 1$, $\mathrm{C} 2\}$ be the subcriteria of $\mathrm{C}$, and let $\left\{\mathrm{C}^{\prime}, \mathrm{C}^{\prime}\right\}$ be of $\mathrm{C}^{\prime}, w, w^{\prime}$ and $w_{i}, w^{\prime}{ }_{i}$ for $i=1,2$ are the corresponding local weights of criteria. Assume all weights of criteria are equal as 0.5 in each group. The total priorities of alternatives, A1 and A2, are also given in Table 4. The global priority vector of alternatives $(\mathrm{A} 1, \mathrm{~A} 2)$ is $(0.488,0.513)$.

Table 4 Example: Rank reversal with criterion addition

\begin{tabular}{|c|c|c|c|c|c|c|}
\hline \multicolumn{7}{|c|}{ Part I: Criteria weights and global sum } \\
\hline $\begin{array}{l}\text { Criteria } \\
\text { Weight }\end{array}$ & & $\begin{array}{l}\mathrm{C} \\
0.5 \\
\end{array}$ & & $\begin{array}{l}C^{\prime} \\
0.5\end{array}$ & & Global Sum \\
\hline $\begin{array}{l}\text { Subcriteria } \\
\text { (Local weight) } \\
\text { Global weight }\end{array}$ & & $\begin{array}{l}\mathrm{C} 1 \\
(0.5) \\
0.25\end{array}$ & $\begin{array}{l}\mathrm{C} 2 \\
(0.5) \\
0.25\end{array}$ & $\begin{array}{l}\text { C'1 } \\
(0.5) \\
0.25\end{array}$ & $\begin{array}{l}C^{\prime} 2 \\
(0.5) \\
0.25\end{array}$ & \\
\hline A1 & & 0.2 & 0.6 & 0.35 & 0.8 & 0.488 \\
\hline A2 & & 0.8 & 0.4 & 0.65 & 0.2 & 0.513 \\
\hline \multicolumn{7}{|c|}{ Part II: Rank reversal with addition of C* } \\
\hline $\begin{array}{l}\text { Subcriteria } \\
\text { (Local weight) } \\
\text { Global weight }\end{array}$ & $\begin{array}{l}\mathbf{C}^{*} \\
(0.5) \\
0.25\end{array}$ & $\begin{array}{l}\mathrm{C} 1 \\
(0.25) \\
0.125\end{array}$ & $\begin{array}{l}\mathrm{C} 2 \\
(0.25) \\
0.125\end{array}$ & $\begin{array}{l}C^{\prime} 1 \\
(0.5) \\
0.25\end{array}$ & $\begin{array}{l}C^{\prime} 2 \\
(0.5) \\
0.25\end{array}$ & \\
\hline A1 & 0.5 & 0.2 & 0.6 & 0.35 & 0.8 & 0.513 \\
\hline A2 & 0.5 & 0.8 & 0.4 & 0.65 & 0.2 & 0.488 \\
\hline
\end{tabular}

Assume that new criterion $\mathrm{C}^{*}\left(w^{*}\right.$, its weight) under $\mathrm{C}$ is added as in Part II of Table 1 . It is also assumed that the weight is equal to the sum of weights regarding $\mathrm{C} 1$ and $\mathrm{C} 2$, and equal output to $\mathrm{A} 1$ and $\mathrm{A} 2$ (indifference). Then, the global priorities of alternatives (A1, A2) change as $(0.513,0.488)$. Finally it is addressed that rank reversal has changed with indifferent criterion added. The problem with this example is related to misunderstanding hierarchic independence.

\subsection{General Steps Using the ANP}

Just as alternatives are instances of a bundle of attributes with respect to which they can be compared, attributes themselves can also be considered as instances of higher order criteria and hence, they can be compared to derive their importance. According to completeness of the expectation axiom, all of the required criteria are included when a decision problem is designed. So, total priorities of alternatives can be expected by the specific evaluation according to each criterion.

Essentially, the outer dependency is based on the condition that each upper criterion covers all necessary sub-criteria. However, if not, addition or deletion of subcriteria effects on the weight of upper criterion and finally on the rank of alternatives. For example as in the above, the added criterion, $\mathrm{C}^{*}$ was assumed to be equivalent to the sum of the previous two criteria priorities, which leads to changes of upper criteria weights. Thus, considering axiom 3 and 4 simultaneously, the upper criterion is impacted by the addition or deletion of lower criteria if it is necessary. Even simple addition of a criterion to a hierarchy also generally requires observation that there is another dependency as a network relationship. If a criterion is added, it is regarded as a new problem to solve. 


\section{Feedback (Reverse Dependency)}

This paper, therefore, takes it general that the addition of new criteria is dealt with by the ANP approach. The effect of new criteria is captured by feedback to criteria or subcriteria as in Figure 1. The supermatrices can represent the input and output of the ANP approaches (See Table 6 and 7). Simple addition of a criterion, $\mathrm{C}^{*}$, generates new local weights vector under criterion $\mathrm{C}$. The new criterion is double the other two previous criteria (See the column of $C$ ).

However, the addition of new criterion affects the normal priorities that show features of contribution to each alternative respectively, even though there is not any additional dependency. In reality the feature vector of criteria to each alternative is obtained by pairwise comparisons. In this paper, we adopt the result of Table 2 for the necessary calculation. If this example is regarded as the allocation of given resources to both projects, A1 and A2 on the same scale, the proportional resource with the amount of a new one to each criterion is summated for each alternative. Then, relative ratio to each criterion is provided for each alternative as in Table 5. As results, the ratio vector of criteria corresponding to the sum of each alternative is calculated as input data in Table 6A (See the column of Al and A2 respectively). If the direction of feedback is to upper criteria, $\mathrm{C}$ and $\mathrm{C}^{\prime}$, input data is the partial sum of subcriteria with regard to A1 and A2 in Table 5 (See the column of A1 and A2 in Table 6B).

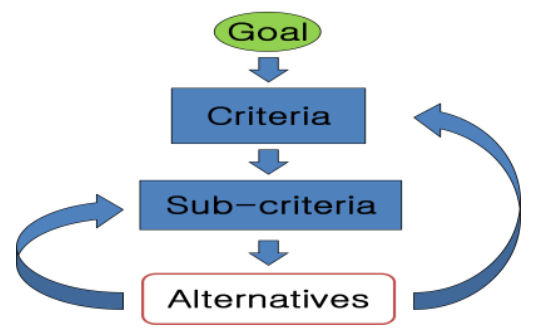

Figure 1 Feedback Direction

Table 5 Features of Criteria for Each Alternative

\begin{tabular}{l|lll|ll|l}
\hline Subcriteria & C* & C1 & C2 & C'1 & C'2 & Sum \\
\hline \multirow{2}{*}{ A1 } & 0.167 & 0.033 & 0.1 & 0.058 & 0.133 & $\mathbf{0 . 4 9 2}$ \\
& $(0.339)$ & $(0.068)$ & $(0.203)$ & $(0.119)$ & $(0.271)$ & \\
\hline \multirow{2}{*}{ A2 } & 0.167 & 0.133 & 0.067 & 0.108 & 0.033 & \multirow{2}{*}{$\mathbf{0 . 5 0 8}$} \\
\hline \multirow{2}{*}{ Total } & $0.328)$ & $(0.262)$ & $(0.131)$ & $(0.213)$ & $(0.066)$ & \\
\hline
\end{tabular}

Ratio to sum is in parenthesis

Table 6A Input: feedback to the second level criteria

\begin{tabular}{l|lll|lllll|ll}
\multicolumn{1}{c}{} & Goal & C & $\mathrm{C}^{\prime}$ & $\mathrm{C}^{*}$ & $\mathrm{C} 1$ & $\mathrm{C} 2$ & $\mathrm{C}^{\prime} 1$ & $\mathrm{C}^{\prime} 2$ & $\mathrm{~A} 1$ & $\mathrm{~A} 2$ \\
\cline { 2 - 11 } Goal & 0 & 0 & 0 & 0 & 0 & 0 & 0 & 0 & 0 & 0 \\
C & 0.5 & 0 & 0 & 0 & 0 & 0 & 0 & 0 & 0 & 0 \\
$\mathrm{C}^{\prime}$ & 0.5 & 0 & 0 & 0 & 0 & 0 & 0 & 0 & 0 & 0 \\
\hline $\mathrm{C}^{*}$ & 0 & 0.5 & 0 & 0 & 0 & 0 & 0 & 0 & 0.339 & 0.328 \\
C1 & 0 & 0.25 & 0 & 0 & 0 & 0 & 0 & 0 & 0.068 & 0.262 \\
C2 & 0 & 0.25 & 0 & 0 & 0 & 0 & 0 & 0 & 0.203 & 0.131 \\
CC$^{\prime} 1$ & 0 & 0 & 0.5 & 0 & 0 & 0 & 0 & 0 & 0.119 & 0.213 \\
CC$^{\prime} 2$ & 0 & 0 & 0.5 & 0 & 0 & 0 & 0 & 0 & 0.271 & 0.066 \\
\hline A1 & 0 & 0 & 0 & 0.5 & 0.2 & 0.6 & 0.35 & 0.8 & 0 & 0 \\
A2 & 0 & 0 & 0 & 0.5 & 0.8 & 0.4 & 0.65 & 0.2 & 0 & 0
\end{tabular}


Table 6B Input: feedback to the first level criteria

\begin{tabular}{|c|c|c|c|c|c|c|c|c|c|c|}
\hline & Goal & C & $C^{\prime}$ & $C^{*}$ & $\mathrm{C} 1$ & $\mathrm{C} 2$ & $C^{\prime} 1$ & $C^{\prime} 2$ & A1 & A2 \\
\hline Goal & 0 & 0 & 0 & 0 & 0 & 0 & 0 & 0 & 0 & 0 \\
\hline $\mathrm{C}$ & 0.5 & 0 & 0 & 0 & 0 & 0 & 0 & 0 & 0.61 & 0.721 \\
\hline $\mathrm{C}^{\prime}$ & 0.5 & 0 & 0 & 0 & 0 & 0 & 0 & 0 & 0.39 & 0.279 \\
\hline $\mathrm{C}^{*}$ & 0 & 0.5 & 0 & 0 & 0 & 0 & 0 & 0 & 0 & 0 \\
\hline $\mathrm{C} 1$ & 0 & 0.25 & 0 & 0 & 0 & 0 & 0 & 0 & 0 & 0 \\
\hline $\mathrm{C} 2$ & 0 & 0.25 & 0 & 0 & 0 & 0 & 0 & 0 & 0 & 0 \\
\hline $\mathrm{C}^{\prime} 1$ & 0 & 0 & 0.5 & 0 & 0 & 0 & 0 & 0 & 0 & 0 \\
\hline $\mathrm{C}^{\prime} 2$ & 0 & 0 & 0.5 & 0 & 0 & 0 & 0 & 0 & 0 & 0 \\
\hline A1 & 0 & 0 & 0 & 0.5 & 0.2 & 0.6 & 0.35 & 0.8 & 0 & 0 \\
\hline A2 & 0 & 0 & 0 & 0.5 & 0.8 & 0.4 & 0.65 & 0.2 & 0 & 0 \\
\hline
\end{tabular}

Table 7 Output from Table 6A and Table 6B

\begin{tabular}{l|ccc|ccccc|cc}
\multicolumn{1}{c}{} & Goal & $C$ & $C^{\prime}$ & $C^{*}$ & $C 1$ & $C 2$ & $C^{\prime} 1$ & $C^{\prime} 2$ & A1 & A2 \\
\cline { 2 - 11 } Goal & 0 & 0 & 0 & 0 & 0 & 0 & 0 & 0 & 0 & 0 \\
C & $(0.667)$ & $(0.667)$ & $(0.667)$ & $(0.667)$ & $(0.667)$ & $(0.667)$ & $(0.667)$ & $(0.667)$ & $(0.667)$ & $(0.667)$ \\
$C^{\prime}$ & $(0.333)$ & $(0.333)$ & $(0.333)$ & $(0.333)$ & $(0.333)$ & $(0.333)$ & $(0.333)$ & $(0.333)$ & $(0.333)$ & $(0.333)$ \\
\hline$C^{*}$ & 0.333 & 0.333 & 0.333 & 0.333 & 0.333 & 0.333 & 0.333 & 0.333 & 0.333 & 0.333 \\
C1 & 0.167 & 0.167 & 0.167 & 0.167 & 0.167 & 0.167 & 0.167 & 0.167 & 0.167 & 0.167 \\
C2 & 0.167 & 0.167 & 0.167 & 0.167 & 0.167 & 0.167 & 0.167 & 0.167 & 0.167 & 0.167 \\
C'1 & 0.167 & 0.167 & 0.167 & 0.167 & 0.167 & 0.167 & 0.167 & 0.167 & 0.167 & 0.167 \\
C'2 & 0.167 & 0.167 & 0.167 & 0.167 & 0.167 & 0.167 & 0.167 & 0.167 & 0.167 & 0.167 \\
\hline A1 & 0.492 & 0.492 & 0.492 & 0.492 & 0.492 & 0.492 & 0.492 & 0.492 & 0.492 & 0.492 \\
A2 & 0.508 & 0.508 & 0.508 & 0.508 & 0.508 & 0.508 & 0.508 & 0.508 & 0.508 & 0.508 \\
\hline
\end{tabular}

The numbers in parentheses (rows of $\mathrm{C}$ and $\mathrm{C}$ ') are only from Table $6 \mathrm{~B}$. They are ' 0 ' from Table $6 \mathrm{~A}$

From the result of Table 7, the final priorities of alternatives (A1, A2) are $(0.492,0.508)$. There is no rank reversal between the two alternatives, A1 and A2 (See Table 1) and total priorities with regards to the alternative coincide with those before adding new but indifferent criterion with the same values of alternatives. Additionally, we can find the weights of $C$ and $C^{\prime}$ as $(0,0)$ from Table $6 \mathrm{~A}$ and as $(0.667$, 0,333 ) from Table $6 \mathrm{~B}$, which means that the weights are transferred to the lower subcriteria. The disappearance is from bidirectional dependency [influence] between five criteria and two alternatives in limiting the supermatrix. The other is from cycle. The result is the same as that of the hierarchic approach in this example.

\section{Conclusion}

Here, it is necessary to note that axiom of the independence or outer dependency of a hierarchy should be viewed with the completeness of the expectation axiom. Its meaning needs to be carefully investigated in reality. When a hierarchy is designed, decomposition process is necessary though the first step is generating and/or collecting criteria. Thus, we can say lower level criteria are outer dependent on a higher criterion, which means the larger concept is gradually decomposed into specific ones until they are 
evaluated or compared obviously. That is, upper criteria are independent from subcriteria in terms of decomposition. However, if there is addition or deletion of any criterion causing rank reversal as argued by previous studies, its composing and designing criteria violate the completeness of the expectation. The axiom of expectation simply says that those thoughtful individuals who have reasons for their beliefs should make sure that their ideas are adequately represented for the outcome to match these expectations, which is based on the fact that all criteria are required to reach such an outcome. This is completeness. If the assumption is violated, it is necessary to redesign the structure of criteria. Then, the given decision making becomes a new problem and generally, ANP is first to be considered. Even though the hierarchical relationship is still kept in the new design, the concept and scale of higher criterion that is impacted by the change of lower criteria need to change. That is, it should investigate how to maintain the expectation hierarchically with the completeness. If the proper way of dealing with such change of criteria is provided like that in this paper considering the two axioms (outer dependency and expectation) simultaneously, then it can be free from irrational/unreasonable rank reversal.

If we consider human being's judgments and decisions, rank reversal is normal and natural, and the intuitional value is different from normatively logical values. For example, jewelry has a high value. Beside the subjective value system, its rareness, that is demand much more than supply, is one of the important items to determine its value even though its objective composition and functional role has not changed. Such evaluation is not normative but descriptive. Finally, the addition of new criteria should be recommended in order to use pairwise comparisons because of judgmental dependency. We also note that rank reversal can occur even with change of independent alternatives but it is natural, for example, with regards to the allocation of given resources or financial support.

\section{REFERENCES}

Finan, J. S. and W. J. Hurley, “The AHP: can wash criteria ignored?, Computer \& Operations Research, 29(2002), 1025-1030.

Millet I. and Saaty, T. L., "On the relativity of relative measures - accommodating both rank preservation and rank reversals in the AHP," European Journal of Operations Research 121 (2000) 201-212

Perez, J., J. L. Jimeno and E. Mokotoff, "Another Potential Shortcoming of AHP," Sociendad de Estadistica e Investigacion Operativa 14/1 (2006),99-111.

Saaty, T. L., "Rank from comparisons and from ratings in the analytic/network processes," European Journal of Operations Research, available online 15 July 2004.

Saaty, T. L., Mathematical Principles of Decision Making, RWS Publication, 2010.

Saaty, T. L. and L. G. Vargas, "The legitimacy of rank reversal," Omega International Journal of Management Science, 12/5(1984), 513-516.

Saaty, T. L. and L. G. Vargas, "The Analytic Hierarchy Process: Wash Criteria Should not Be Ignored, International Journal of Management and Decision Making, Vol. 7, Nos.2/3, 2006

Saaty, T. L., L. G. Vargas and R. Whitaker, "Addressing with Brevity Criticisms of the Analytic Hierarchy Process, IJAHP (2009) 1/2, 121-134 